\title{
Acetyl Coenzyme A
}

National Cancer Institute

\section{Source}

National Cancer Institute. Acetyl Coenzyme A. NCI Thesaurus. Code C199.

The condensation product of coenzyme $A$ and acetic acid which participates in the biosynthesis of fatty acids and sterols, in the oxidation of fatty acids and in the metabolism of many amino acids. In addition, Acetyl Coenzyme A acts as a biological acetylating agent. 